\title{
Bilateral Asymmetry Detection in Digital Mammography Using B-spline Interpolation
}

\author{
Jelena Bozek, Emil Dumic and Mislav Grgic \\ University of Zagreb Faculty of Electrical Engineering and Computing \\ Department of Wireless Communications \\ Unska 3/XII, 10000 Zagreb, Croatia \\ jbozek@ieee.org
}

\begin{abstract}
Digital mammography allows detection of breast abnormalities in early stage of breast cancer development. One of the abnormalities that may indicate breast cancer in its early stage is bilateral asymmetry. This paper presents computer-aided detection algorithm for bilateral asymmetry that uses B-spline interpolation for breast alignment. Alignment of the right and left breast is important step in computer-aided detection algorithm in order to allow comparison of corresponding points in right and left breast. Differential analysis of breasts is based on simple subtraction technique. The results are highlighted with color in each image and presented on a computer monitor thereby indicating radiologist the regions that need to have a second look and be further investigated. We found that our approach can be a very useful tool for radiologists in detection and diagnosis of bilateral asymmetry.
\end{abstract}

Keywords - digital mammography; bilateral asymmetry; breast alignment; interpolation; computer-aided detection

\section{INTRODUCTION}

Digital mammography is one of the best examination methods for detecting breast cancer in early stage of development and thereby increasing chances for patient's complete recovery. It can reveal pronounced evidence of abnormality, such as masses and calcifications, as well as subtle signs such as bilateral asymmetry and architectural distortion [1]. Bilateral asymmetry, i.e. asymmetry of the breast parenchyma between left and right breast, may indicate breast cancer in its early stage. According to ACR's (American College of Radiology) Breast Imaging Reporting and Data System [2] there are two types of bilateral asymmetry: global asymmetry and focal asymmetry. Global asymmetry is defined when a greater volume of fibroglandular tissue is present in one breast compared to the corresponding area in the other breast and focal asymmetry is circumscribed area of asymmetry seen on two views, but it lacks the borders and conspicuity of a mass. Focal asymmetry is usually an island of healthy fibroglandular tissue that is superimposed with surrounding fatty tissue. Bilateral asymmetries of concern are those that are changing or enlarging or new, those that are palpable and those that are associated with other findings, such as microcalcifications or architectural distortion [3].
Humans are susceptible to committing errors and their analysis is usually subjective and qualitative. Objective and quantitative analysis facilitated by the application of computers to biomedical image analysis leads to a more accurate diagnostic decision by the physician [4]. Computer-aided detection algorithms have been developed to aid radiologists in finding the suspicious breast abnormalities and set up the accurate diagnosis [5].

This paper presents a method for bilateral asymmetry detection in which the left and right breasts were aligned using the B-spline interpolation [6]. After the breast alignment the differential analysis was performed. The difference between the breasts was calculated using simple subtraction technique. The resulting right and left images containing differences that were highlighted with green and blue color, respectively, were displayed in Matlab using the algorithm presented in [7].

This paper is organized as follows. In Section II some previous work in bilateral asymmetry detection is summarized. The proposed method that uses B-spline interpolation and subtraction for bilateral asymmetry detection is described in Section III. Section IV presents the results of the proposed method and Section $\mathrm{V}$ draws the conclusions and gives directions for possible future research.

\section{PREVIOUS WORK}

There are not many algorithms developed for bilateral asymmetry detection. It may be due to the fact that the first step in bilateral asymmetry detection is a challenging task. The first step that has to be performed before comparing images is some kind of registration or alignment of the breasts. Alignment procedures have to confront many difficult problems such as the natural asymmetry of the breasts, absence of good corresponding points between left and right breast images to perform matching and distortions inherent to breast imaging [8]. Georgsson [9] proposed method for bilateral registration based on anatomical features and assumptions of how the female breast is deformed under compression. He established an anatomical coordinate system defined with pectoral muscle and nipple. The coordinate system was used to divide breast into two boxes containing upper and lower part of the breast. 
He independently scaled each of these boxes to match the bilateral counterpart in size and transformed the skin lines of the two breasts to match each other. For differential analysis he proposed two methods. The first method was based on the absolute difference between the registered images and the second method was based on the statistical differences between properties of corresponding neighborhoods.

Some authors proposed techniques for bilateral asymmetry detection that do not involve any procedure for the registration or alignment of the breasts. Ferrari et al. [8] presented a procedure based upon the detection of linear directional components. They used a multiresolution representation based on Gabor wavelets. The Gabor filter responses for different scales and orientation were analyzed by using Karhunen-Loeve (KL) transform and Otsu's method of thresholding. For quantitative and qualitative analysis of the oriented patterns the rose diagrams were used. Rangayyan et al. [10] extended the method of Ferrari et al. [8] by including morphological measures and geometric moments of the fibroglandular disk related to density distributions. Directional features were obtained from the difference rose diagrams and additional set of features including Hu's moments, eccentricity, stretch, area and average density were extracted from the segmented fibroglandular disks. The differences between the pairs of the features for the left and right mammographic image were used as measures for the analysis of asymmetry. They concluded that morphological features and moments of the fibroglandular disks, used on their own or in combination with directional features, were not effective in the recognition of bilateral asymmetry.

\section{BILATERAL ASYMMETRY DETECTION}

The first step in bilateral asymmetry detection is alignment of the breasts. Alignment ensures that the correct corresponding points of the left and right breast are being compared. Original right and left MLO (mediolateral oblique) images are shown in Figure 1.

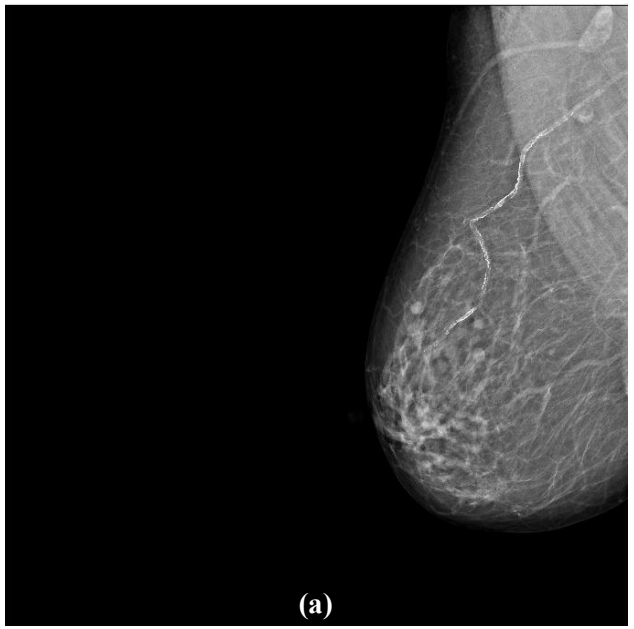

Figure 1. Original MLO images of the: (a) right breast, (b) left breast
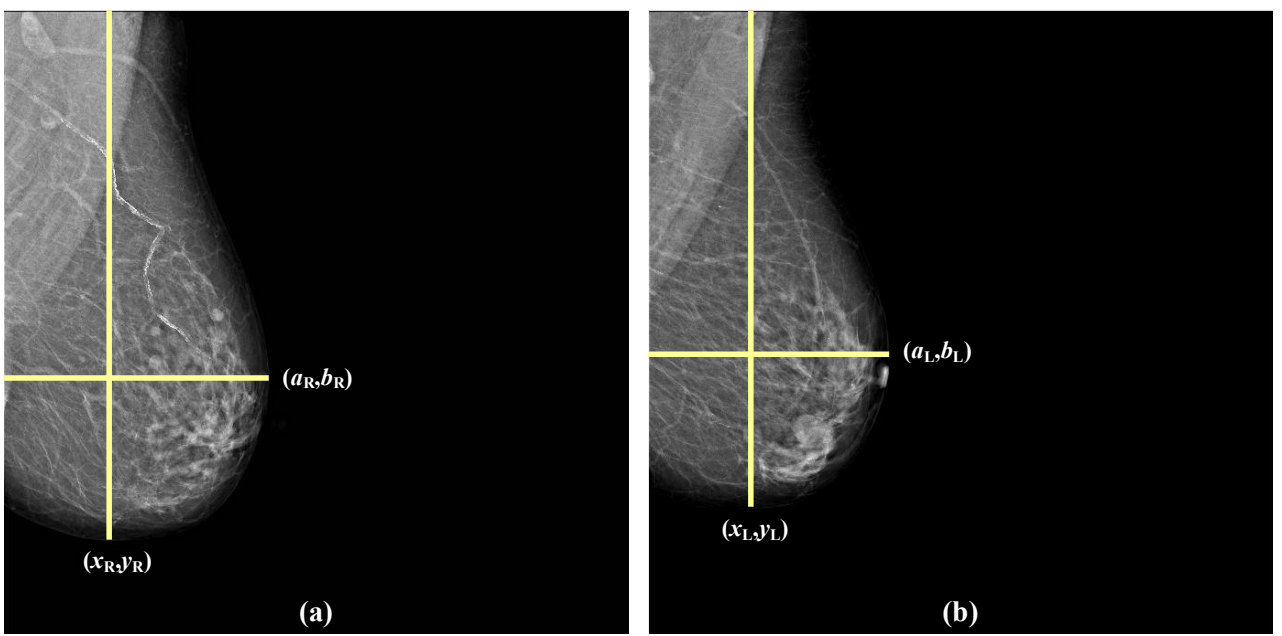

Figure 2. Coordinates of the furthest points on the breast contour in the:

(a) flipped right image, (b) left image 
Before the alignment right breast image is flipped so that the breasts have the same orientation thereby making the alignment process simpler. The proposed alignment method is based on the B-spline interpolation [6]. Image interpolation is a method of constructing new data points within the range of a discrete set of known data points. With B-spline interpolation interpolated pixels (picture elements) are computed using sixteen nearest known samples (four in horizontal by four in vertical direction) [11]. For the interpolation procedure the size ratio of left and right breast has to be found. In order to find the size ratio between the left and right breast the furthest point of the breast contour from the image edge is found for each breast. The coordinate system with marked furthest points in horizontal and vertical direction is shown in Figure 2. Coordinate points named $\left(a_{\mathrm{R}}, b_{\mathrm{R}}\right)$ and $\left(a_{\mathrm{L}}, b_{\mathrm{L}}\right)$ are the furthest points in the horizontal direction of the right and left breast, respectively. Coordinate points named $\left(x_{R}, y_{R}\right)$ and $\left(x_{L}, y_{L}\right)$ are the furthest points in the vertical direction of the right and left breast, respectively.

New image size $h$ in the vertical direction that is defined by the interpolation scale factor $x_{\mathrm{L}} / x_{\mathrm{R}}$ is calculated as follows:

$$
h=\left(x_{\mathrm{L}} / x_{\mathrm{R}}\right) \cdot m,
$$

where $m$ is the number of rows in original mammographic image. New image size $w$ in the horizontal direction that is defined by the interpolation scale factor $b_{\mathrm{L}} / b_{\mathrm{R}}$ is calculated as follows:

$$
w=\left(b_{\mathrm{L}} / b_{\mathrm{R}}\right) \cdot n,
$$

where $n$ is the number of columns in original mammographic image.

B-spline interpolation implemented in Matlab was used for interpolating flipped right breast to the size of the left breast according to the scale factors. Using described interpolation we achieved the required alignment of the breasts and it is now possible to compare their corresponding regions. The difference between two breasts is computed by subtraction of interpolated right breast image and left breast image.

For better display and visualization of differences between left and right breast on the computer monitor and to indicate regions that need to be further investigated by radiologist, the differences in each breast are displayed with different colors. The regions in interpolated right breast that differ from the corresponding regions in left breast are highlighted with green color and the regions in left breast that differ from the corresponding regions in interpolated right breast are highlighted with blue color.

\section{EXPERIMENTAL RESULTS}

Algorithm proposed in this paper is tested on a set of 70 pairs of right and left MLO digital mammographic images, from 70 different patients. Images are provided from the digital mammographic image database developed in cooperation with the Department of Diagnostic and Interventional Radiology, University Hospital "Dubrava", Zagreb, Croatia. Each image in our database has resolution of $4084 \times 3328$ pixels with 12 bits per pixel. The image format is uncompressed TIFF. All images are stored in compliance with Dicom standard. Mammographic imaging device is Mammomat NovationDR that has $\alpha$ Selenium direct conversion detector and uses Wolfram/Rhodium anode/filter combination [12]. Images are displayed in Matlab using the algorithm that automatically adjusts contrast and brightness, regardless of bit depth of an image, number of amplitudes higher than zero and the number of allocated bits [7]. We would like to point out that our proposed algorithm for alignment of the breasts and bilateral asymmetry detection works well with nonsegmented images and there is no need for additional preprocessing and segmentation step.

Result of the proposed method is shown in Figure 3. Figure 3(a) and (b) show the original flipped right and original left digital mammographic images, respectively. After finding the furthest points on the breast contour that are shown in Figure 2 and described in previous Section, the flipped right breast image is interpolated using the B-spline interpolation method that is implemented in Matlab. Flipped and interpolated right breast image shown in Figure 3(c) is aligned with the original left image shown in Figure 3(d). When the breasts are aligned it is possible to compute differences between them. The absolute difference between flipped and interpolated right breast image and original left breast image is shown in Figure 3(e). For better illustration and visualization of possible abnormalities, regions in breast that differ it from contralateral breast are colored. Difference that occurs in flipped and interpolated right breast compared to the left breast is highlighted with green and showed in Figure 3(f). Difference that occurs in left breast compared to the flipped and interpolated right breast is highlighted with blue and showed in Figure 3(g).

\section{CONCLUSION}

First step in bilateral asymmetry detection in mammographic images is the alignment of the right and left breast. In this paper we propose a B-spline interpolation method for breast alignment that shows good results. Colored display of regions with differences in left and right images helps radiologist to more precisely inspect the suspicious region. In future work we plan to make a thorough comparative analysis of the use of different interpolation methods in digital mammography as well as develop a new method for differential analysis of bilateral asymmetry based on correlation between the regions of interest in mammographic images.

\section{ACKNOWLEDGMENT}

The work in this paper was conducted under the research project "Intelligent Image Features Extraction in Knowledge Discovery Systems" (036-0982560-1643), supported by the Ministry of Science, Education and Sports of the Republic of Croatia.

The authors would like to thank Prof. Boris Brljacic, Dr. Renata Huzjan-Korunic and Mr. Milan Grzan from the Department of Diagnostic and Interventional Radiology, University Hospital "Dubrava", Zagreb, Croatia, for providing digital mammographic images. 

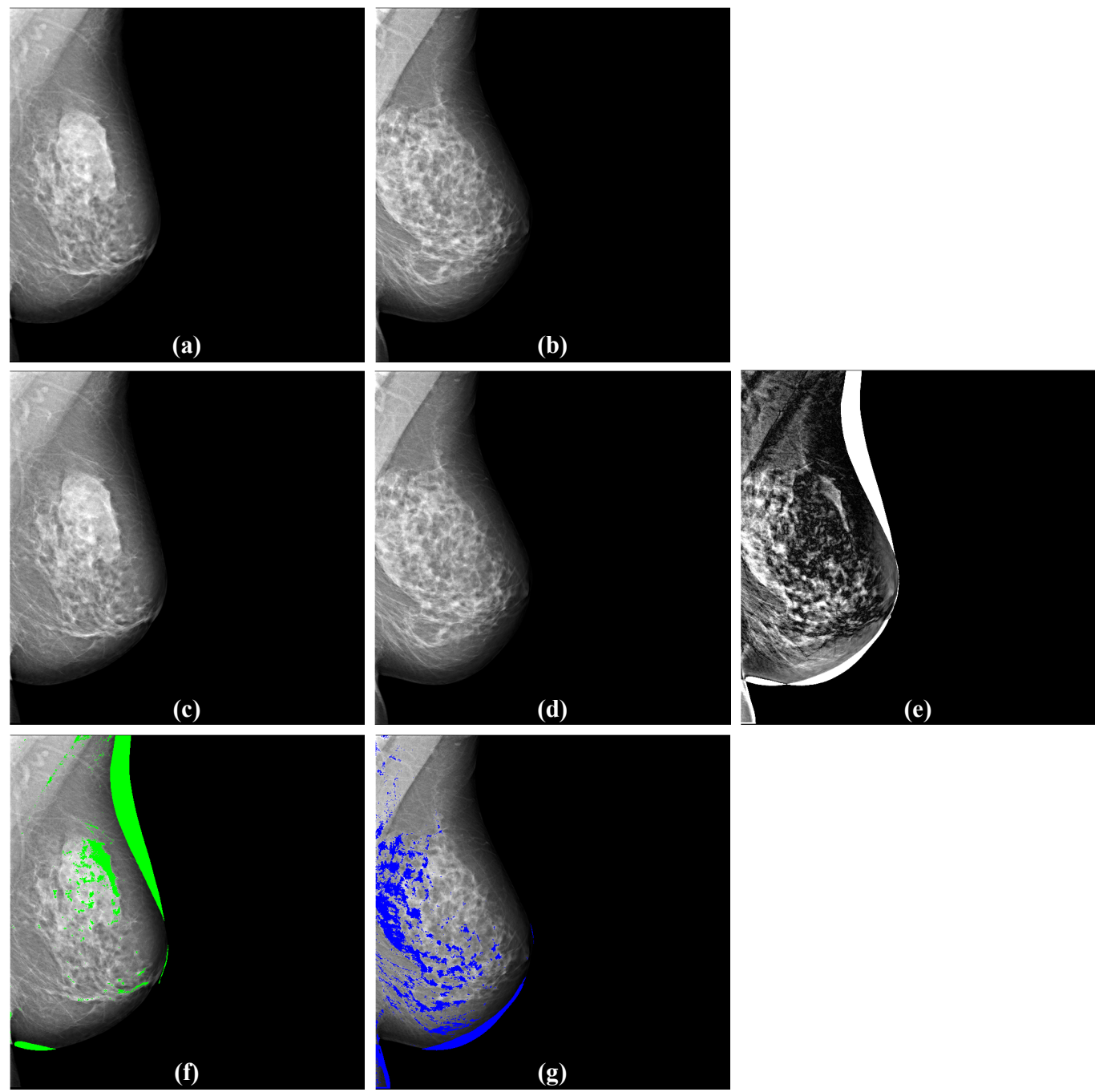

Figure 3. Results of the bilateral asymmetry detection algorithm: (a) flipped original right breast image, (b) original left breast image, (c) flipped and interpolated right breast image, (d) original left breast image, (e) absolute difference between flipped and interpolated right breast image and left breast image, (f) difference in flipped and interpolated right breast image compared to the left breast image, $(\mathrm{g})$ difference in left breast image compared to the flipped and interpolated right breast image

\section{REFERENCES}

[1] M. J. Homer, Mammographic Interpretation: A Practical Approach, 2nd edition, McGraw-Hill, New York, 1997

[2] American College of Radiology (ACR): ACR Breast Imaging Reporting and Data System, Breast Imaging Atlas, 4th edition, Reston, VA, USA, 2003

[3] E. S. de Paredes, Atlas of Mammography, 3rd edition, Lippincott Williams \& Wilkins, Philadelphia, USA, 2007

[4] R. M. Rangayyan: Biomedical Image Analysis, CRC Press LLC, Boca Raton, Florida, USA, 2005

[5] Mammographic Image Analysis Homepage, www.mammoimage.org

[6] P. Thevenaz, T. Blu, M. Unser, "Image Interpolation and Resampling", in Handbook of Medical Imaging, Processing and Analysis, Academic Press, 2000, pp. 393-420

[7] M. Mustra, M. Grgic, and K. Delac, "Efficient Presentation of DICOM Mammography Images using Matlab", Proceedings of the 15th International Conference on Systems, Signals and Image Processing (IWSSIP), Bratislava, Slovakia, pp. 13-16, 25-28 June 2008
[8] R. J. Ferrari, R. M. Rangayyan, J. E. L. Desautels, and A. F. Frere, "Analysis of asymmetry in mammograms via directional filtering with Gabor wavelets", IEEE Trans. Med. Imag., Vol. 20, pp. 953-964, September 2001

[9] F. Georgsson, "Differential analysis of bilateral mammograms", in Int. J. Pattern Recognition and Artificial Intelligence, Vol. 17, pp. 1207-1226, July 2003

[10] R. M. Rangayyan, R. J. Ferrari, and A. F. Frere, "Analysis of bilateral asymmetry in mammograms using directional, morphological, and density features", Journal of Electronic Imaging, Vol. 16, Jan-Mar 2007

[11] E. Dumic, S. Grgic, and M. Grgic, "Hidden Influences on Image Quality when Comparing Interpolation Methods", Proceedings of the 15th International Conference on Systems, Signals and Image Processing (IWSSIP), Bratislava, Slovakia, pp. 367-372, 25-28 June 2008

[12] Siemens, Mammomat NovationDR, Available at: www.medical.siemens.com 